\title{
Effects of Alprazolam Administration on the Vital Organs of Adult Wister Albino Rats, Biochemical and Toxicological Studies
}

\author{
Atiskumar Chattopadhyay, ${ }^{1, \star}$ Soumendra Darbar², Srimoyee Saha ${ }^{3}$, Parimal Karmakar ${ }^{4}$ \\ ${ }^{1}$ Principal Secretary, Faculty Council of Science, Jadavpur University 188, Raja S C Mallick Road Kolkata-700032, West Bengal, INDIA. \\ ${ }^{2}$ Bioequivalence Study Centre, Department of Pharmaceutical Technology, Jadavpur University, Kolkata 700 032, West Bengal, INDIA. \\ ${ }^{3}$ Department of Physics, Jadavpur University, Kolkata 700 032, West Bengal, INDIA. \\ ${ }^{4}$ Department of Life Science and Biotechnology, Jadavpur University, 188, Raja S.C. Mullick Road, Kolkata 700 032, West Bengal, INDIA.
}

\begin{abstract}
Objective: Effect of Benzodiazepine group of drugs Alprazolam is investigated on different vital organs of Wister albino rat. Materials and Methods: The rats $(n=6)$ were treated with normal doses of Alprazolam for two months along with a placebo group $(n=6)$. After the treatment blood samples were collected and then the rats were sacrificed to collect the tissues of heart, liver and kidney. Results: The levels of enzymes ALT/SGPT, AST/SGOT and the signaling molecule NO were estimated. It was seen that the levels of all these three parameters were increased in all the treated rats. NO is a very important chemical messenger and cellular signaling molecule. Increased level of NO in the tissues of vital organs can modulate the normal physiological and cellular processes and homeostasis of the body in many ways. The NO mediated macrophage activation may also be influenced by the Benzodiazepine (BDZ) group of drugs. So, it's a very important finding from the clinical point of view. Prolonged use of Alprazolam shows different contraindications and affects different vital organs and systems of the body. To know how the drug affects heart, liver and kidney the levels of two clinically important enzymes like ALT/ SGPT and AST/ SGOT were measured in serum and tissue extracts of rat. These two enzymes are very good indicator of cardiac and hepatic health. Results of the experiments show that levels of both the enzymes were increased in serum and tissue extracts, particularly in the tissue extract of heart. Conclusion: So, from our above studies it can be concluded that there is a relation between increase of $\mathrm{NO}$ and the increase of levels of clinically important enzymes. As a result, before frequent and prolonged administration of the drug Alprazolam, further detailed study is needed regarding its effect on different vital organs and cellular and physiological processes.
\end{abstract}

Key words: Alprazolam (Alp), Cell death, Necrosis, Biochemical enzymes, Nitric Oxide (NO).

\section{INTRODUCTION}

In the recent decade, there has been increasing concern on the hazardous effects of drugs on different species. Benzodiazepines (BDZ) comprise a large group of psychoactive drugs that are massively used in human pharmaco-therapy for their anxiolytic and hypnotic anticonvulsant properties. ${ }^{1}$ Alprazolam (Alp) is a member of BDZs group of drugs, widely used as antipsychotic and anxiolytic agent. They are used for the acute and sub-chronic treatment of insomnia and agitated psychosis. ${ }^{2}$ Once it was considered as a safe drug altogether. But for a decade or long reports are coming that Alprazolam can damage the vital organ like heart, liver, kidney etc.

A vast amount of studies have shown that alprazolam in doses of $0.5 \mathrm{mg}$ and higher induce changes in brain oxidative metabolism and impairs a variety of cognitive and psychomotor skills such as memory impairment, speed of responses, tracking
Submission Date: 20-06-2018; Revision Date: 14-08-2018; Accepted Date: 23-10-2018

DOI: 10.5530/ijper.53.1.17 Correspondence: Dr. Atiskumar Chattopadhyay,

Principle Secretary, Faculty Council of Science, Jadavpur University 188 ,

Raja S C Mallick Road Kolkata-700032,

West Bengal, INDIA. Phone no: +919038044928 E-mail: atischatterjee@gmail. com

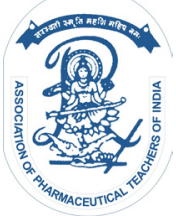

www.ijper.org 
performance and a considerable potential to induce dependence and abuse. ${ }^{3-5}$ Moreover, scientific study established that Alp produce conformational changes in hemoglobin ${ }^{6}$ and it also intercalates into the DNA. ${ }^{7}$ Tanaka and its co workers reported that some popular drinks may exert cytotoxic effects in combination with BDZs, for example, Alp and ethanol administration in together had toxicity in liver microsomes in vitro. ${ }^{8}$

Research revealed that Intravenous administration of Alp caused an acute significant decrease in brain activation as well as in the whole-brain cerebral blood flow of $25 \%$ to $30 \%$. ${ }^{9}$ Verster et al. concluded that driving is unsafe after administration of Alp because of it produces adverse effects such as drowsiness and sedation, in addition to its therapeutic effect. ${ }^{10,11}$

In addition, it was found that alprazolam produced severe cytotoxicity in human cell lines as it induced elevated level of reactive oxygen species (ROS) and depletion of antioxidants. ${ }^{12}$ Generally, it was reported that all benzodiazepines drugs can cause respiratory depression ${ }^{13}$ hepatic dysfunction ${ }^{14}$ hypotension and thrombophlebitis, ${ }^{15,16}$ brain damage. ${ }^{17}$ It is also observed that alprazolam caused cytotoxicity in rat vital organs. Yousif et. al. studies on mice stated that Alprazolam at a dose of $0.2 \mathrm{mg} / \mathrm{kg}$ body weight observed extensive morphological changes like enlargement of the nuclei and proliferation of Kuffer cells in the liver cells. ${ }^{18}$

Alprazolam has lots of toxic and adverse side effects on persons under long term treatment with this drug. Recent study showed, ${ }^{19}$ alprazolam was found to induce a significant increase in neutrophil count and a significant decrease in lymphocytes, anti-SRBC titer and IL-2 level with severe depletion of the splenic, thymal and nodal lymphocytes, accompanied by congestion and eosinophilic vasculitis of all vital organs.

So, objective of the study is to establish the potential toxic effects of alprazolam on the several blood enzymes level like Alanine aminotransferase (ALT)/ serum glutamate pyruvate transaminase (SGPT), Aspartate aminotransferase (AST)/ serum glutamate oxaloacetate transaminase (SGOT) in rat serum and tissue extract of heart, liver and kidney as well as to measure the NO levels.

\section{MATERIALS AND METHODS Drugs and Chemicals}

Alprazolam powder was procured from Torrent Pharmaceutical Ltd, India and was dissolved in MiliQ water with $0.24(\mathrm{~N}) \mathrm{HCl}$ solution through vigorous vortexing. The dose was calculated $0.2 \mathrm{mg} / 100 \mathrm{gm}$ body weight/ day. ${ }^{18}$ All chemical reagents were of analytical grades purchased from Sigma Chemical Co. (St. Louis, Mo, USA), Merk (Germany) and BDH (England).

\section{Animals}

Three months old albino male rats (100-120gm) were obtained from National Institute of Hydrabad (CPCSEA approval No. 154/99/CPCSEA) Animals were housed in large size polyethylene cages under standard hygienic condition. All rat were acclimatized to the laboratory environment for two weeks prior to the starting of the experiment, where they were adapted to the controlled environmental conditions at room temperature of $25 \pm 2{ }^{\circ} \mathrm{C}$, relative humidity $60-70 \%$ and at normal photoperiod $12 \mathrm{~h} / \mathrm{d}$. The methodology of this work was approved by the Institutional Animal Ethics Committee (IAEC) on Animal. All animals were fasted for 1 hour prior to drug administration.

\section{Experimental Design}

For treatments with alprazolam rats were divided into two groups in either sex: normal healthy group (group 1) and drug (Alp) treated group (group 2), each of 6 rats. Alprazolam drug was administered intraperitonially using a single dose of $0.2 \mathrm{mg} / 100 \mathrm{gm}$ body weight/day.

\section{Monitoring of the Signs of Toxicity}

All rats of the experiment were closely observed and carefully examined daily throughout the experimental period in order to see whether any apparent behavioural changes and/or signs of toxicity are present or not.

\section{Body Weights and Different Organs Weights}

Body weights of all experimental rats were recorded weekly during the period of treatment. Means of the body weights and body weight gains were estimated. At the end of the experiment, both control and experimental groups were sacrificed and dissected. The organs were excised out quickly, weighed and the absolute and the relative weights were calculated according to Matousek (1969) I.W. = organ weight $(\mathrm{g}) / 100 \times$ body weight (g).

\section{Collection of Sample and Biochemical Study}

After 60 days blood samples were collected at the end of the experiment via cardiac puncture from each anaesthetized rat (Ketamin hydrochloride with Xylazin) after fasting 8-12 h, using disposable syringes. Samples were centrifuged at $3500 \mathrm{rpm}$ for $15 \mathrm{~min}$ and then the clear serum was collected in sterilized disposable plastic tubes and stored in a freezer set at $-20^{\circ} \mathrm{C}$ for subsequent measurement of serum biochemical parameters.

Serum ALT and AST were measured by colorimetric method by using commercially available standard 
diagnostic Kit (Spain diagnostic limited, India). The above parameters were also measured from tissue extract of liver, heart and kidney. At the end of experiments, rats were sacrificed Under controlled anesthesia and the liver, kidney and heart samples were collected, minced and homogenized in either ice cold distilled water using a glass homogenizer. The homogenates were centrifuged for $15 \mathrm{~min}$ at $10000 \mathrm{~g}$. at 4 degree $\mathrm{C}$ and the supernatants and was used for different biochemical analysis.

\section{Determination of NO by Griess Method}

The total nitrite was determined in the tissue extracts according to the standard biochemical protocol of Greiss method as a measure of NO.

\section{Statistical Analysis}

Statistical analysis was done using the ANOVA and test for comparison of data in the control group with the experimental groups. The results were expressed as mean \pm S.E.M (standard error of means). P-value less than 0.05 were considered significant and are written in the parentheses.

\section{RESULTS}

\section{Effect of Alprazolam on Signs of Toxicity}

The results revealed prominent signs of toxicity in rats administered with $0.2 \mathrm{mg} / 100 \mathrm{~g}$ alprazolam (ALP), where most treated animals became progressively less active and showed general weakness. We also noticed that they had lost their appetite during and after the experiment of treatment with the drug and noticed marked loss in their body sizes. Control rats showed no mortality during the experimental period, however, there was few mortality observed in ALP-treated rats.

\section{Effect of Alprazolam on Body Weight Change}

At the end of the experiment, rats treated with ALP showed marked significant decreases in the body weight change, comparing to the control (Table 1).

\section{Effect of Alprazolam on Relative Different Organ Weight}

In this study statistically significant difference in organ weight of the liver, kidneys and heart were found by the intoxication of alprazolam compared to untreated animals. The liver, kidneys and heart were considerably lighter in the mice from the group exposed to Alprazolam (ALP) compared to the rats from the control group (Table 2 ).

\section{Effect of Alprazolam on Serum and Tissue Protein and Enzyme Estimation}

The serum level of total protein and levels of ALT and AST in serum and tissue extracts of liver, heart and kidney in experimental animals showed on (Table 3 and 4). However, the levels of ALT and AST in serum were all significantly higher in alprazolam treated

\begin{tabular}{|c|c|c|c|c|}
\hline \multicolumn{5}{|c|}{ Table 1: Effect of Alprazolum on Body Weight of rat. } \\
\hline Group & Initial Body Weight (g) & Final Body weight (g) & Net Gain (g) & Average Food Intake (g) \\
\hline Control & 122.56 & 152.89 & $30.33 \pm 4.32$ & $13.68 \pm 1.72$ \\
\hline Treated & 122.52 & 136.41 & $13.89 \pm 2.14$ & $15.21 \pm 1.49^{* * *}$ \\
\hline
\end{tabular}

All values represent the mean \pm SEM. P values calculated by ANOVA followed by Dunnett's post hoc test of significance. ${ }^{*} p<0.05$ as compared with Control, ${ }^{* *} p<0.01$ as compared with control, $* * * P<0.001$ as compared with control

\begin{tabular}{|c|c|c|c|}
\hline \multicolumn{4}{|c|}{ Table 2: Effect of Alprazolum on Wet Weight of Different Organ of rat. } \\
\hline Group & Heart (g) & Liver (g) & Kidney (g) \\
\hline Control & $2.86 \pm 0.22$ & $6.45 \pm 0.66$ & $4.22 \pm 0.06$ \\
\hline Treated & $2.74 \pm 0.34$ & $7.56 \pm 0.67^{\star * *}$ & $4.18 \pm 0.05^{\star \star *}$ \\
\hline
\end{tabular}

All values represent the mean \pm SEM. P values calculated by ANOVA followed by Dunnett's post hoc test of significance. ${ }^{*} p<0.05$ as compared with Control, ${ }^{* *} p<0.01$ as compared with control, $* * * P<0.001$ as compared with control

\begin{tabular}{|c|c|c|c|c|}
\hline \multicolumn{7}{|c|}{ Table 3: ALT (IU/L) in serum and tissue extract of rat. } \\
\hline Group & Serum (IU/L) & Heart (IU/L) & Liver (IU/L) & Kidney (IU/L \\
\hline Control & $51.84 \pm 2.76$ & $145.33 \pm 9.72$ & $190.17 \pm 8.82$ & $48.17 \pm 1.15$ \\
\hline Treated & $62 \pm 6.68$ & $426.33 \pm 22.86^{\star * *}$ & $265.17 \pm 15.54^{\star *}$ & $169.167 \pm 3.2^{\star * *}$ \\
\hline
\end{tabular}

All values represent the mean \pm SEM. P values calculated by ANOVA followed by Dunnett's post hoc test of significance. ${ }^{*} p<0.05$ as compared with Control, ${ }^{* *} p<0.01$ as compared with control, $* * * \mathrm{P}<0.001$ as compared with control. 


\begin{tabular}{|c|c|c|c|c|}
\hline \multicolumn{7}{|c|}{ Table 4: AST (IU/L) in serum and tissue extract of rat. } \\
\hline Group & Serum (IU/L) & Heart (IU/L) & Liver (IU/L) & Kidney (IU/L \\
\hline Control & $211.84 \pm 11.86$ & $271 \pm 17.2$ & $182.3 \pm 4.86$ & $154.16 \pm 9.22$ \\
\hline Treated & $246.34 \pm 14.20$ & $352.17 \pm 20.22^{* *}$ & $229.83 \pm 8.66^{\star *}$ & $290.5 \pm 16.46$ \\
\hline
\end{tabular}

All values represent the mean \pm SEM. P values calculated by ANOVA followed by Dunnett's post hoc test of significance. ${ }^{*} p<0.05$ as compared with Control, ${ }^{* *} p<0.01$ as compared with control, $* * * P<0.001$ as compared with control.

animals when compared to control values. The increases of the above two enzyme concentrations were also noted in the liver, kidneys and heart in comparison with control group.

\section{Effect of Alprazolam on Tissue NO Concentration}

The effects of alprazolam on tissue NO levels (in terms of total nitrite concentrations) were given in Figure 1-3. Alp. treatment caused a significant increase in total nitrite concentration at a dose of $0.2 \mathrm{mg} / 100 \mathrm{~g}$ alprazolum compared with control group in the tissue extracts of heart, liver and kidney (Figure 1-3).

\section{Concentration of Nitrite In Tissue Extract of Heart} (in $\mu \mathrm{M}$ )

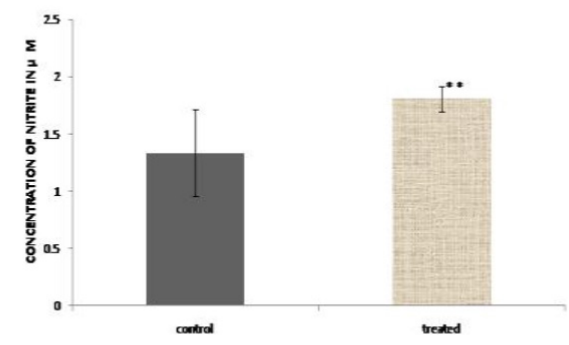

All values represent the mean $₫$ SEM. Pvahes calculated by ANOVA followed by Dunnett's post hoc test of significance

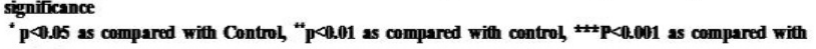
control

Figure 1: Estimation of total nitrite in rat heart tissue extract after treatment of Alprazolam as compared with control animals.

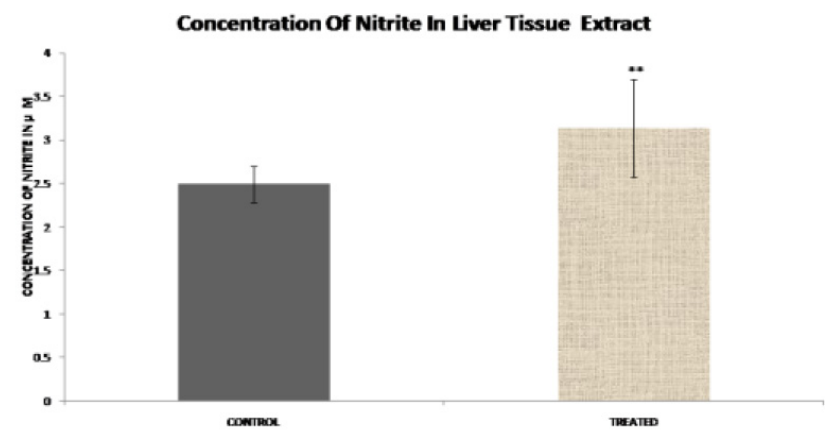

All vahes represent the mean \pm SEM. P values cakculated by ANOVA followed by Dunnetił post hoc test of significance

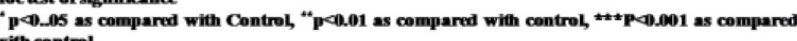

Figure 2: Estimation of total nitrite in rat liver tissue extract after treatment of Alprazolam as compared with control animals.

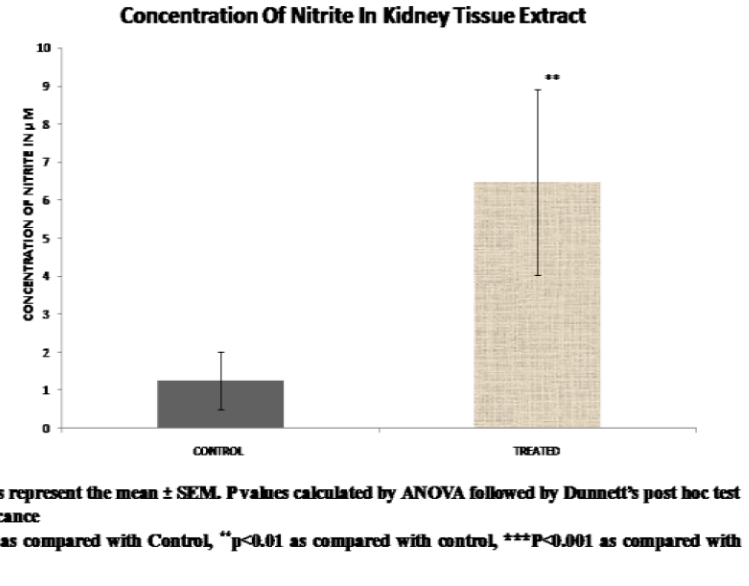

Figure 3: Estimation of total nitrite in rat kidney tissue extract after treatment of Alprazolam as compared with control animals.

\section{DISCUSSION}

Alprazolam is highly prescribed sedative drug used in the treatment of anxiety disorders. It is readily absorbed in intestinal tract. After absorption, the drug is transported through blood to different tissues. In central nervous system the drug binds with the receptor, which is a GABA receptor. ${ }^{20}$ As a result chloride channel at the membrane of the neuron opens. This causes the hyperpolarisation of the membrane. Thus, depolarization of the membrane or stimulation of the neuron becomes difficult. In this process NO acts as a mediator. But in other tissues also, there are PBR. The drug binds with that receptor also. So, the objective of the study was to see whether the binding of drug alprazolam with PBR increases the level of $\mathrm{NO}$ in other tissues and blood also. ${ }^{21}$ From the result of the study it has been seen that NO level increased in the tissue extracts of the vital organs like heart, liver and kidney of rat after prolonged administration of Alprazolam. NO is a very important chemical messenger and cellular signaling molecule. Increased level of $\mathrm{NO}$ in the tissues of vital organs can modulate the normal physiological process and homeostasis of the body in many ways. ${ }^{22}$ So, it's a very important finding from the clinical point of view. The NO mediated macrophage activation may also be influenced by the BDZ group of drugs. Further studies can be done to trace the mechanism of generation of $\mathrm{NO}$ and it's effects on important physiological processes. 
Prolonged use of Alprazolam shows different contraindications and affects different vital organs and systems of the body. To know how the drug affects heart, liver and kidney the levels of two clinically important enzymes like ALT and AST were measured in serum and tissue extracts of rat. These two enzymes are very good indicator of cardiac and hepatic health. Results of the experiments shows that levels of both the enzymes increased in serum and tissue extracts, particularly in the tissue extract of heart. These are the important and also novel findings of the study.

So, from the study it can be concluded that before frequent and prolonged administration of Alprazolam detailed and further study is needed regarding its effect on different vital organs and cellular and physiological processes.

\section{ACKNOWLEDGEMENT}

The authors are thankful to University Grant Commission, New Delhi, India for providing financial grants. [F.PSW074/05-06(ERO) dated 21.03.2006].

\section{CONFLICT OF INTEREST}

Authors have none to declare

\section{ABBREVIATIONS}

Alp: Alprazolam; ALT: Alanine Aminotransferase; AST: Aspartate Aminotransferase; BDZ: Benzodiazepine; CPCSEA: Committee for the Purpose of Control and Supervision of Experiments on Animals; GABA: GammaAminobutyric Acid; IAEC: Institutional Animal Ethics Committee; IL-2: Interleukin-2; NO: Nitric Oxide; PBR: Peripheral benzodiazepine receptor; ROS: Reactive Oxygen Species; SGOT: Serum Glutamate Oxaloacetate Transaminase; SGPT: Serum Glutamate Pyruvate Transaminase; SRBC: Sheep Red Blood Cells.

\section{REFERENCES}

1. Mandrioli R, Mercolini L, Raggi MA. Benzodiazepine metabolism: an analytical perspective. Curr Drug Metab. 2008;9(8):827-44.

2. Charney, DS, Heninger GR, Jatlow PI. Increased anxiogenic effects of caffeine in panic disorders. Archives of General Psychiatry. 1985;42(3);233-43.

3. Verster JC, Volkerts ER. Antihistamines and driving ability: evidence from on-the-road driving studies during normal traffic. Ann Allergy Asthma Immunol. 2004;92(3):294-3.
4. Pardo HG, Conejo NM, Arias JL. Oxidative metabolism of limbic structures after acute administration of diazepam, alprazolam and zolpidem. Prog Neuro-Psychopharmacol Biol Psychiatry. 2006;30(6):1020-6.

5. Leufkens TRM, Vermeeren A, Smink BE, Ruitenbeek PV, Ramaekers JG Cognitive, psychomotor and actual driving performance in healthy volunteers after immediate and extended release formulations of alprazolam $1 \mathrm{mg}$ Psychopharmacol. 2007;191(4):951-59.

6. Maitra S, Saha B, Santra CR, Mukherjee A, Goswami S, Chanda PK et al. Alprazolam induced conformational change in hemoglobin. Int $\mathrm{J}$ Biol Macromolecules. 2007;41(1):23-9.

7. Saha B, Mukherjee A, Santra CR, Chattopadhyay A, Ghosh AN, Choudhuri U, Karmakar P. Alprazolam intercalates into DNA. J Biomolecul Structure Dynamics. 2009;26(4): 421-9.

8. Tanaka E, Nakamura T, Terada M, Shinozuka T, Honda K. Metabolic interaction between ethanol, high-dose alprazolam and its two main metabolites using human liver microsomes in vitro. J Forensic Leg Med. 2007;14(6):348-51.

9. Deborah MD, Cowley S, Peter P, Roy-Byrne, Allen R, James C, et al. Benzodiazepine sensitivity in panic disorder: Effects of chronic alprazolam treatment. Neuropsychopharmacology. 1995;12 (2):147-7.

10. Verster JC, Volkerts ER, Schreuder AH, Eijken EJ, Heuckelum JHV, Veldhuijzen DS, et al. Residual effects of middle-of-the-night administration of zaleplon and zolpidem on driving ability, memory functions and psychomotor performance. J Clin Psychopharmacol. 2002;22(6):576-83.

11. Verster JC, Volkerts ER, Verbaten MN. Effects of alprazolam on driving ability, memory functioning and psychomotor performance: a randomized, placebo-controlled study. Neuropsychopharmacology. 2011;27(2):260-9.

12. Saha B, Mukherjee A, Samanta S, Saha P, Ghosh AK, Santra CR, et al. Caffeine augments Alprazolam induced cytotoxicity in human cell lines. Toxicol in vitro. 2009;23(6):1100-9.

13. Woodcock AA, Gross ER, Geddes DM. Drug treatment of breathlessness: contrasting effects of diazepam and promethazine in pink puffers. BMJ (Clin Res Ed). 1981;283(6287):343-6.

14. Ananth J, Swartz R, Burgoyne K, Gadasally R. Hepatic disease and psychiatric illness: relationships and treatment. Psychother Psychosom. 1994;62(3-4):146-59.

15. Donaldson D, Gibson G. Systemic complications with intravenous diazepam. Oral Surg Oral Med Oral Pathol. 1980;49(2):126-30.

16. Glaser JW, Blanton PL, Thrash WJ. Incidence and extent of venous sequelae with intravenous diazepam utilizing a standardized conscious sedation technique. J Periodontol. 1982;53(11):700-3.

17. Meador KJ. Cognitive side effects of antiepileptic drugs. Can J Neurol Sci. 1994;21(3):S12-6.

18. Yousif B, Wafaa A. Microscopic Studies on the Effect of Alprazolum (Xanax) on the Liver of Mice. Pakistan Journal of Biological Sciences. 2002;5(11):1220-25

19. Ghada E, Elmesallamy M, Abass A, Nahla AG, Ahmed R, Amal HA Differential effects of alprazolam and clonazepam on the immune system and blood vessels of non-stressed and stressed adult male albino rats. Interdiscip Toxicol. 2011;4(3):132-43.

20. Judd FK, Norman TR, Marriott PF, Burrows GD. A case of alprazolam-related hepatitis. Am J Psychiatry. 1986;143:388-9.

21. Roy-Byrne P, Vittone BJ, Uhde TW. Alprazolam-related hepatotoxicity. Lancet. 1983;2:786-7.

22. Reith DM, Fountain J, McDowell R, Tilyard M. Comparison of the fatal toxicity index of zopiclone with benzodiazepines. J Toxicol Clin Toxicol. 2003;41(7):975-80. 


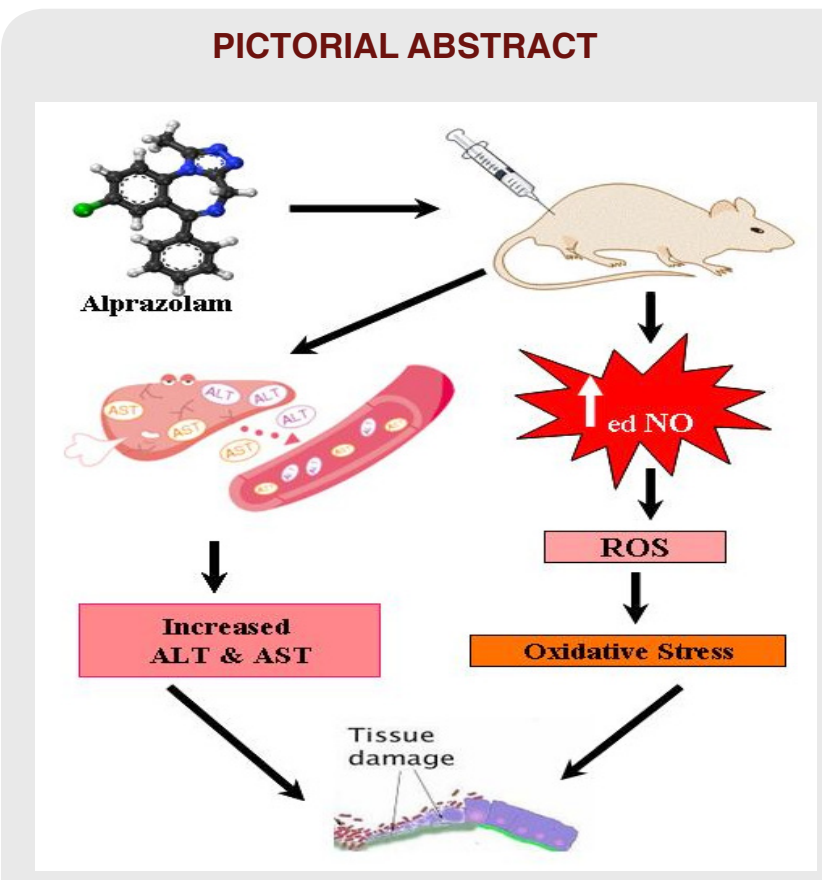

About Authors
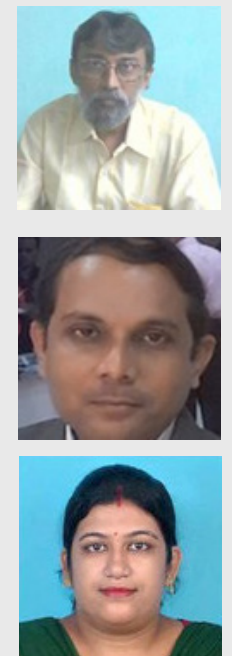

Atiskumar Chattopadhyay, Principal Secretary, Faculty Council of Science, Jadavpur University, 188, Raja S.C. Mullick Road, Kolkata 700 032, West Bengal, India

Soumendra Darbar, Research Scientist, Bioequivalence Study Centre, Department of Pharmaceutical Technology, Jadavpur University, Kolkata 700 032, West Bengal, India

Srimoyee Saha, Research Scholar, Department of Physics, Jadavpur University, Kolkata 700 032, West Bengal, India

Parimal Karmakar, Professor, Department of Life Science and Biotechnology, Jadavpur University, 188, Raja S.C. Mullick Road, Kolkata 700 032, West Bengal, India

Cite this article: Chattopadhyay A, Darbar S, Saha S, Karmakar P. Effects of Alprazolam Administration on the Vital Organs of Adult Wister Albino Rats, Biochemical and Toxicological Studies. Indian J of Pharmaceutical Education and Research. 2019;53(1):127-32. 